\title{
Systemic and rapidly progressive light-chain deposition disease initially presenting as tubulointerstitial nephritis
}

\author{
Satoko Takahashi $\cdot$ Jun Soma Izaya Nakaya Mayumi Yahata \\ Tsutomu Sakuma · Hiroshi Yaegashi · Akiyoshi Sato • Masaharu Wano • \\ Hiroshi Sato
}

Received: 14 January 2012/ Accepted: 10 June 2012/Published online: 19 July 2012

(C) Japanese Society of Nephrology 2012

\begin{abstract}
A 42-year-old woman was admitted to a hospital after first-time detection of proteinuria and hematuria during a routine medical check-up. Because her serum creatinine level had rapidly increased from 0.9 to $3.2 \mathrm{mg} / \mathrm{dl}$ since measurement 3 months earlier, she was referred to our hospital. Renal biopsy revealed extensive tubular atrophy and interstitial fibrosis with mild leukocyte infiltration. Glomeruli showed minimal changes, and no immunoglobulin or complement deposition was observed by immunofluorescence. Oral prednisolone was commenced under the diagnosis of chronic tubulointerstitial nephritis, and she discharged once. However, its effects were transient; her renal function deteriorated rapidly and hemodialysis was initiated 5 months after her initial checkup. On readmission, urinary Bence-Jones protein $\kappa$-type was detected, and examination of bone marrow led to a diagnosis of Bence-Jones $\kappa$-type multiple myeloma. Light-
\end{abstract}

S. Takahashi · J. Soma $(\bowtie) \cdot$ I. Nakaya $\cdot$ M. Yahata

Division of Nephrology, Iwate Prefectural Central Hospital,

1-4-1 Ueda, Morioka, Iwate 020-0066, Japan

e-mail: sjun@chuo-hp.jp

S. Takahashi

e-mail: kikuiritaka@yahoo.co.jp

T. Sakuma $\cdot$ H. Yaegashi

Division of Pathology, Iwate Prefectural Central Hospital,

Morioka, Iwate 020-0066, Japan

A. Sato $\cdot$ M. Wano

Division of Hematology, Iwate Prefectural Central Hospital,

Morioka, Iwate 020-0066, Japan

H. Sato

Department of Clinical Pharmacology and Therapeutics, Tohoku University Graduate School of Pharmaceutical Sciences and Medicine, Tohoku University, Sendai,

Miyagi 980-8578, Japan chain staining using a renal biopsy specimen obtained 2 months earlier showed $\kappa$-light-chain deposition on tubular basement membranes but not glomeruli. Despite undergoing chemotherapy with vincristine, doxirubicin, and dexamethasone, the patient died suddenly from a cardiac arrhythmia. Autopsy showed $\kappa$-light-chain deposition in the heart, thyroid, liver, lungs, spleen, and ovaries. Congo red staining yielded negative results. Typical lightchain deposition disease (LCDD) characterized by nodular glomerulosclerosis was observed in the kidneys. This case demonstrates that tubulointerstitial nephritis can be an early pathological variant of LCDD, which may be followed by accelerated and massive light-chain deposition in glomeruli.

Keywords Tubulointerstitial nephritis - Monoclonal immunoglobulin deposition disease $\cdot$ Cardiac death . Cardiac arrhythmia - Light-chain deposition disease . Multiple myeloma

\section{Introduction}

Light-chain deposition disease (LCDD) is one of three entities (LCDD, light and heavy chain deposition disease, and heavy chain deposition disease) of non-amyloidotic monoclonal immunoglobulin deposition diseases (MIDD) $[1,2]$. They are histopathologically characterized by nodular glomerulosclerosis, resembling nodular diabetic glomerulosclerosis, idiopathic nodular glomerulosclerosis, and nodular membranoproliferative glomerulonephritis, and deposition of monoclonal immunoglobulin components in glomeruli and tubular basement membranes [1-3]. Most patients with MIDD present with proteinuria or nephrotic syndrome, with or without renal dysfunction [2]. Of the 
MIDD, LCDD shows the highest incidence, commonly affecting the kidneys. The extent to which light-chain deposition occurs in organs other than the kidney is unknown; however, other organ involvement is considered common [4].

In LCDD, tubular casts composed of light chains coprecipitated with Tamm-Horsfall protein obstruct distal tubules, causing intense interstitial inflammation and acute renal failure [5]. This phenomenon is termed light-chain cast nephropathy. However, acute tubulointerstitial nephritis accompanied by neither cast nephropathy nor glomerular lesions has been recently reported in patients with plasma cell dyscrasias [6].

Here, we report the case of a patient initially diagnosed with chronic tubulointerstitial nephritis without casts or glomerular lesions on renal biopsy. The patient developed end-stage renal failure 2 months after initial renal biopsy and died because of cardiac arrhythmia after the diagnosis of LCDD with multiple myeloma and initiation of chemotherapy.

\section{Case report}

A 42-year-old woman with no significant medical history consulted her primary physician in May 2010 after proteinuria and hematuria were identified during a routine medical check-up. The patient was followed up by the primary physician, but she began to experience anorexia and the general malaise associated with an increase in serum creatinine from 0.9 to $3.2 \mathrm{mg} / \mathrm{dl}$ over a period of 3 months. She was referred and admitted to our hospital in early August 2010. On admission, her blood pressure was $125 / 78 \mathrm{mmHg}$, her height was $152 \mathrm{~cm}$, and her weight was $53 \mathrm{~kg}$. Physical examination was unremarkable except for mild lower extremity edema. Urinalysis revealed proteinuria of $2.0 \mathrm{~g} /$ day and 50-99 red blood cells/high-power field, with only hyaline casts. Her laboratory findings were as follows: serum creatinine level, $3.5 \mathrm{mg} / \mathrm{dl}$; serum total protein level, $6.9 \mathrm{~g} / \mathrm{dl}$; serum albumin level, $4.2 \mathrm{~g} / \mathrm{dl}$; serum sodium level, $138 \mathrm{mEq} / \mathrm{l}$; serum potassium level, $4.4 \mathrm{mEq} / \mathrm{l}$; serum chloride level, $104 \mathrm{mEq} / \mathrm{l}$; serum calcium level, $10.3 \mathrm{mg} / \mathrm{dl}$; serum phosphorus level, $5.0 \mathrm{mg} / \mathrm{dl}$; urinary $\alpha 1$-microglobulin excretion, $20500 \mu \mathrm{g} / 1$ (normal $500-9000 \mu \mathrm{g} / \mathrm{l}) ; \quad$ urine $N$-acetyl- $\beta$-D-glucosaminidase, $7.9 \mathrm{U} / \mathrm{l}$ (normal $<5 \mathrm{U} / \mathrm{l}$ ); urinary sodium concentration, $60.0 \mathrm{mEq} / \mathrm{l}$; urinary potassium concentration, $30.9 \mathrm{mEq} / \mathrm{l}$; FENa, $2.7 \%$; leukocyte count, $10.5 \times 10^{3} / \mu \mathrm{l}$; erythrocyte count, $5.03 \times 10^{6} / \mu \mathrm{l}$; hemoglobin level, $15.0 \mathrm{~g} / \mathrm{dl}$; hematocrit, $42.2 \%$; platelet count, $291 \times 10^{3} / \mu \mathrm{l}$; blood urea nitrogen level, $29.6 \mathrm{mg} / \mathrm{dl}$; uric acid level, $4.0 \mathrm{mg} / \mathrm{dl}$; total cholesterol level, $162 \mathrm{mg} / \mathrm{dl}$; triglyceride level, $153 \mathrm{mg} / \mathrm{dl}$; C-reactive protein level, $0.1 \mathrm{mg} / \mathrm{dl}$; serum IgG level,
$1409 \mathrm{mg} / \mathrm{dl}$ (normal 870-1700 mg/dl); serum IgA level, $131 \mathrm{mg} / \mathrm{dl}$ (normal 110-410 mg/dl); and serum IgM level, $96 \mathrm{mg} / \mathrm{dl}$ (normal 34-220 mg/dl). Her serum C3 and C4 levels, total serum hemolytic activity (CH50), hepatobiliary function, and blood glucose levels were normal. Tests for anti-myeloperoxidase and proteinase-3 neutrophil antibodies, anti-nuclear antibody, anti-double-stranded DNA antibody, rheumatoid factor, and cryoglobulins were also negative. Serum protein electrophoresis on cellulose acetate did not show M-peak, but serum and urine immunoelectrophoresis were not done. Computed tomography of the abdomen revealed swelling of the kidneys bilaterally, whereas electrocardiography showed no abnormalities. Percutaneous renal biopsy was performed for accurate diagnosis.

Nineteen glomeruli were examined microscopically; almost half were globally sclerotic, with the remaining glomeruli showing a slight increase in the mesangial matrix without mesangial proliferation or capillary-wall abnormalities. No crescents were observed. Tubular atrophy and interstitial fibrotic changes were notable, along with dilatation of some tubules, mild infiltration of mononuclear cells, and very few tubular casts (Fig. 1a-c). Immunofluorescence for $\operatorname{IgG}, \operatorname{Ig} \mathrm{A}, \operatorname{IgM}, \mathrm{C} 1 \mathrm{q}, \mathrm{C} 3$, and fibrinogen was negative. Electron microscopy was not performed. The findings were consistent with those of chronic tubulointerstitial nephritis. No drug abuse was evident, and we ruled out diseases that cause secondary tubulointerstitial nephritis, such as Sjögren's syndrome, sarcoidosis, and gout. Then, we diagnosed the patient with idiopathic chronic tubulointerstitial nephritis and administered oral prednisolone $(40 \mathrm{mg} /$ day $)$ after methylprednisolone pulse therapy $(500 \mathrm{mg} /$ day for three consecutive days). At discharge (middle of September 2010), the patient was on $25 \mathrm{mg} /$ day oral prednisolone and her serum creatinine level, urinary protein excretion level, and urinary red blood cell count were $3.5 \mathrm{mg} / \mathrm{dl}, 0.4 \mathrm{~g} / \mathrm{day}$, and 20-29/highpower field, respectively.

However, as an outpatient, her serum creatinine level increased again, and she was readmitted to our hospital in the middle of October 2010. On readmission, her serum creatinine level was $7.3 \mathrm{mg} / \mathrm{dl}$ and she showed marked abnormalities of the immunoglobulin profile that were undetected during the first admission: serum $\operatorname{IgG}$ level, $408 \mathrm{mg} / \mathrm{dl}$; serum IgA level, $42 \mathrm{mg} / \mathrm{dl}$; and serum IgM level, $46 \mathrm{mg} / \mathrm{dl}$. Plasma cell dyscrasia was suspected for the first time. Thus, we performed serum and urinary immunoelectrophoresis and bone marrow examination. Urine and serum positivities for Bence-Jones protein- $\kappa$ and an increase in bone marrow plasma cells $(31.4 \%)$ indicated Bence-Jones $\kappa$-type multiple myeloma. Light-chain staining of a renal biopsy specimen obtained during the first admission showed $\kappa$-light-chain deposition on tubular 

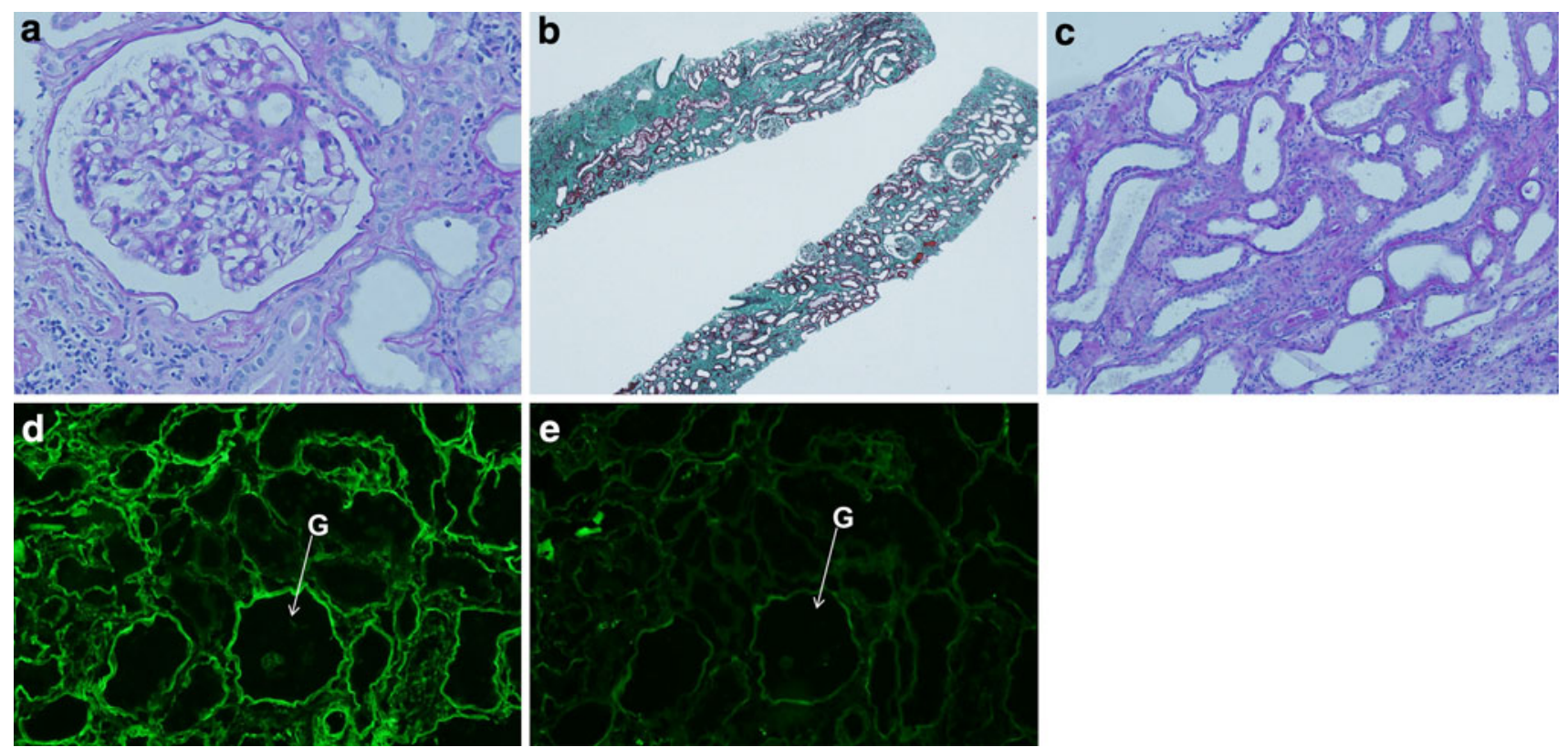

Fig. 1 Renal biopsy findings. a No increase in the mesangial matrix or mesangial proliferation was observed. Tubular atrophy and interstitial fibrosis associated with mild mononuclear cell infiltration were evident around the glomerulus. No definite abnormalities of the capillary walls were observed. b Tubular atrophy and increase of the interstitial space were observed in more than half of the tubulointerstitial area, with mild infiltration of mononuclear cells and few casts.
Mild dilation of some tubules was also seen. c Atrophic and dilated tubules with simplified flattened epithelium and thickened tubular basement membranes were observed. d Staining for $\kappa$-light chains was positive along tubular basement membranes but was negative in glomeruli. e $\lambda$-light-chain deposition was not observed. a, c Periodic acid-Schiff stain and b Elastica-Masson stain. $\mathbf{a} \times 200, \mathbf{b} \times 20$, c-e $\times 100 . G$ glomerulus
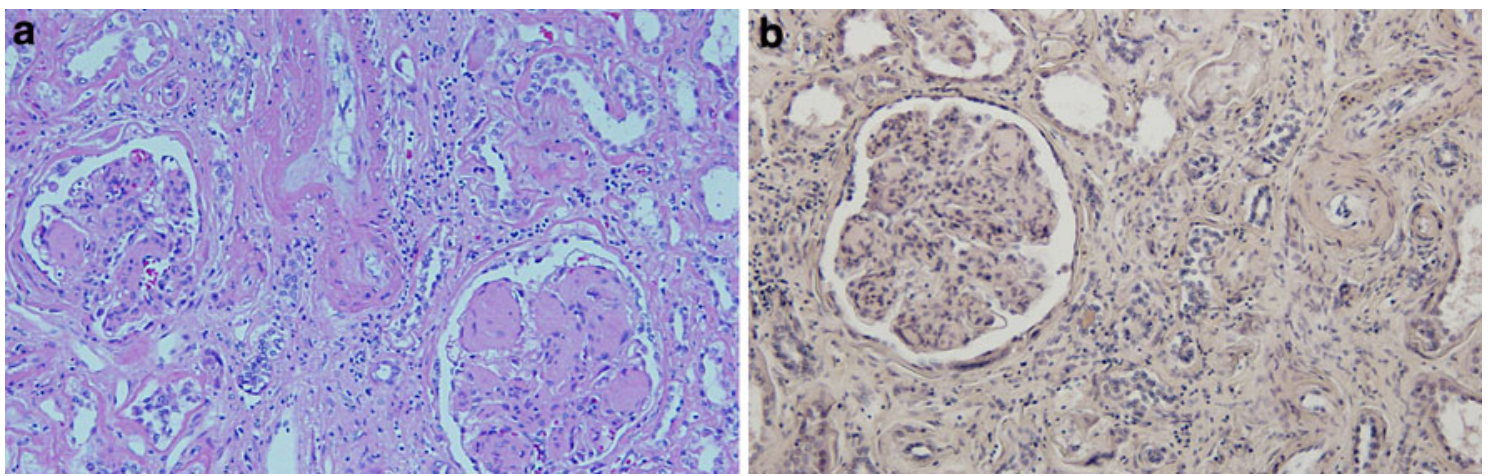

Fig. 2 Autopsy findings of the kidneys. a Nodular glomerulosclerosis was clearly visible. The structure of tubules was destroyed, compatible with marked interstitial fibrosis. b Congo red staining on glomeruli, tubules, and vessels yielded negative results. a H\&E stain. a, b $\times 100$

basement membranes with no definite deposition on glomeruli and vessels (Fig. 1d). $\lambda$-light-chain (Fig. 1e) and Congo red staining yielded negative results. These findings led us to a final diagnosis of tubulointerstitial nephritis associated with LCDD. Hemodialysis was initiated soon after admission because she experienced dyspnea and pulmonary congestion, which was confirmed by chest $\mathrm{X}$-ray. Chemotherapy with vincristine, doxirubicin, and dexamethasone (VAD) was commenced in late November 2010 for treating multiple myeloma. Hemodialysis improved her general condition. Holter electrocardiography showed monofocal premature ventricular contractions, but this was not considered dangerous. However, the patient died suddenly from cardiac arrhythmia in early December 2010.

The autopsy showed $\kappa$-light-chain deposition in the heart (cardiac muscle as well as atrioventricular and sinoatrial nodes), thyroid, liver, lungs, spleen, and ovaries, in addition to the kidneys. In the kidneys, all glomeruli had nodular sclerotic lesions, characteristic of LCDD (Fig. 2a), which was not observed in the renal biopsy specimen. Severe tubular atrophy and interstitial fibrosis were diffusely observed. Congo red staining of the kidneys (Fig. 2b) and all other organs yielded negative results. 

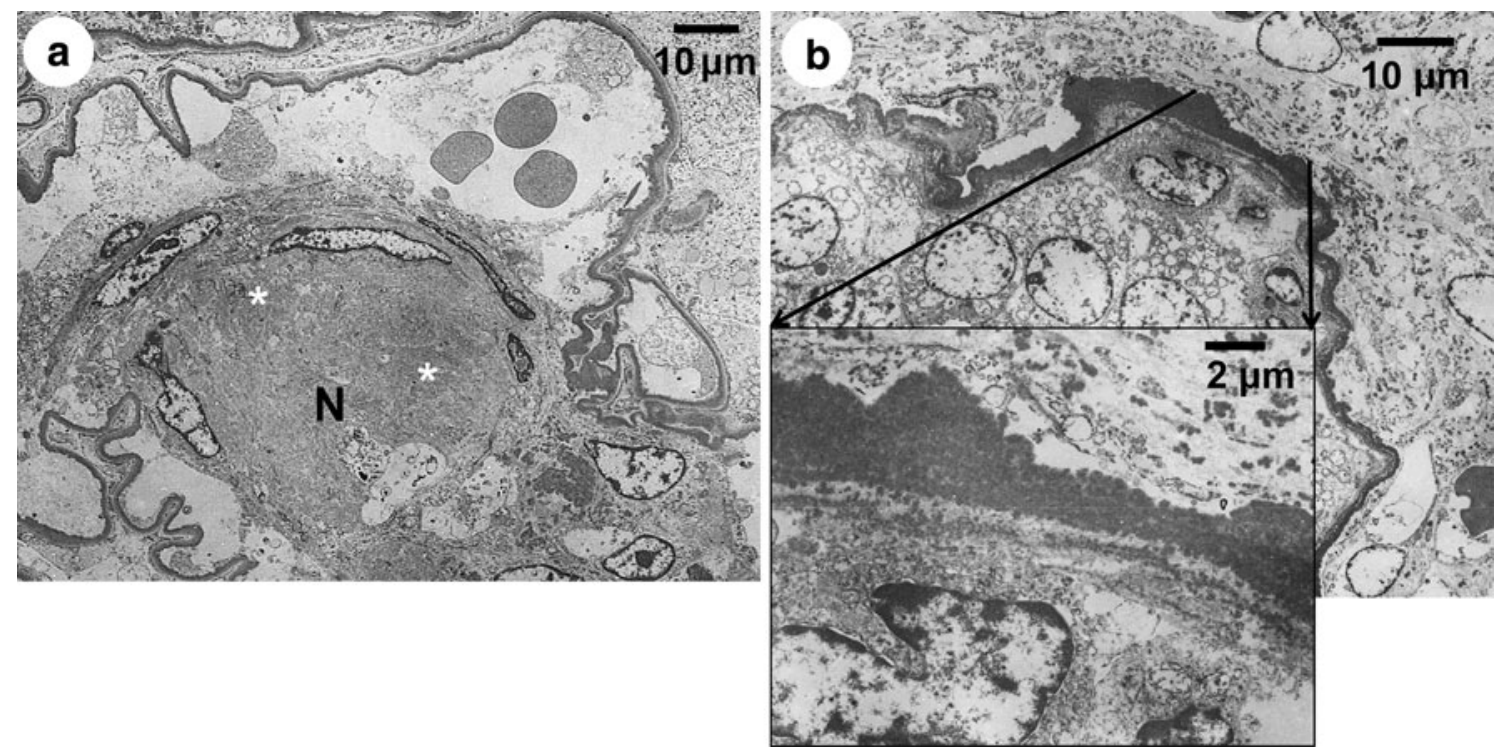

Fig. 3 Electron microscopy findings. a Electron-dense deposits in the inner aspect of the glomerular basement membrane and in the mesangial nodule were seen. However, mesangial electron-dense deposits (asterisks) were subtle or finely granular. b Electron-dense

Electron microscopy showed granular electron-dense deposits in the inner aspect of glomerular basement membranes (Fig. 3a). Electron-dense deposits in nodular mesangial lesions were also observed, but at a lower density than that in glomerular basement membranes. Deposits in tubular basement membranes were highly dense and tended to be concentrated on their outer aspect (Fig. 3b).

\section{Discussion}

Monoclonal immunoglobulin deposition diseases, including LCDD, are characterized by the deposition of nonamyloidotic monoclonal immunoglobulin components in glomeruli and tubular basement membranes that induce nodular glomerulosclerosis, along with glomerular and tubular basement membrane thickening [1-3]. However, it was recently reported that LCDD with tubulointerstitial nephritis is poorly recognized in patients with plasma cell dyscrasia [6]. The pattern had no glomerular lesions on light microscopy, no specific staining being performed for light chains on glomeruli and vessels on immunohistochemistry, and no casts or multinucleated giant cells causing secondary tubulointerstitial changes being observed-the so-called "cast nephropathy" [5]. Histopathological findings from a renal biopsy specimen in the present case were considered to be corresponded to the poorly recognized pattern of renal disease associated with LCDD. In addition, this case showed dilatation of some tubules with flattened epithelium, which is one of the deposits were observed along the tubular basement membrane. The highly dense deposits were located on the outer aspect of the basement membrane and were heavily concentrated. $N$ nodular glomerular lesion

characteristics suggesting acute tubular injury [7]. Therefore, it was likely that acute tubular injuries influenced, at least in part, the rapid deterioration of renal function.

The present case should be distinguished from immunoglobulin light-chain proximal tubulopathy (LCPT) [812] because some cases of LCPT show interstitial fibrosis without apparent glomerular lesions on light microscopy [8]. Immunofluorescence study of LCPT demonstrates light-chain restriction limited to proximal tubular epithelium, and electron microcopy demonstrates rhomboid crystals or phagolysosomes in the proximal tubular epithelium [8-12]. Most cases of LCPT were reported to be associated with Fanconi syndrome [9-12]. In the present case, no light-chain depositions were observed within the proximal tubular epithelium on immunofluorescence study. Rhomboid crystals or phagolysosomes were not found under detailed observations of proximal tubules by electron microscopy. Laboratory data at the time of renal biopsy did not show hypophosphatemia, hypokalemia, hyperchloremia, or glucosuria, which suggests Fanconi syndrome. Therefore, the present case could be diagnosed with tubulointerstitial nephritis associated with LCDD.

Apart from eight cases reported by Gu and Herrera [6], a few case reports have documented light-chain-associated tubulointerstitial nephritis with myeloma, without cast nephropathy and glomerular or vascular lesions [13, 14]. All cases, including the present case, presented with acute renal failure or a rapidly progressive clinical course, and 10 out of 11 cases showed $\kappa$-light chains in the serum and/or urine. Among these cases, this is the first case report 
describing light-chain-associated tubulointerstitial nephritis followed by rapid and massive light-chain deposition in glomeruli. The exact mechanisms of the development of tubulointerstitial nephritis remain unclear. Light chains, reaching tubular basement membranes via peritubular capillaries, might alter intrinsic tissue antigens, resulting in cytokine release, which induces chemoattraction and activation of interstitial mononuclear inflammatory cells [6]. However, inflammatory cell infiltration was scarce in this case, suggesting that another mechanism was responsible. One possible explanation is that our patient was at a chronic stage of tubulointerstitial nephritis; hence, the deposition of light chains on tubular basement membranes decreased or stopped, and light-chain deposition started in glomeruli. However, how the deposition is regulated is uncertain. Moreover, this case showed a rapidly progressive course; nodular glomerular lesions characteristic of LCDD were formed during a period of 2-3 months. These observations demonstrate that accelerated and massive deposition of light chains in glomeruli occurred following light-chain deposition on tubular basement membranes.

Initially, LCDD was reported to be a systemic disorder characterized by monoclonal light-chain deposition in systemic organs [15]. Patient survival varies from a few months to 10 years, and depends on extrarenal light-chain deposition, especially in the heart $[2,16,17]$. In this case, $\kappa$-light chains were observed in cardiac muscle as well as in atrioventricular and sinoatrial nodes. Renal failure is common and rapidly progresses to end-stage renal failure in most patients with LCDD, regardless of early chemotherapy [2, 17]. A recent retrospective study of 11 patients $<65$ years of age with LCDD or light and heavy chain deposition disease who were treated by high-dose chemotherapy with the support of autologous peripheral blood stem cell transplantation (PBSCT) documented no treatment-related death and a decrease in monoclonal immunoglobulin levels in eight patients. In addition, complete disappearance of monoclonal immunoglobulin from serum and urine was observed in six patients, in whom improvement in manifestations related to deposits and histological regression of light-chain deposition in cardiac, hepatic, and skin biopsies were documented [18]. Furthermore, resolution of nodular glomerular lesions [19] and reversal of dialysis-dependence renal failure and sustained improvement in renal function [20] have also been reported. However, all studies of high-dose therapy with PBSCT support in patients with LCDD were performed retrospectively in small numbers of patients. Whether this therapy provides benefits compared with conventional chemotherapy remains to be established.

A key step in the diagnosis of MIDD is immunofluorescence analysis of the kidney. Unfortunately, routine immunofluorescence in our laboratory did not include $\kappa$ - and $\lambda$-light-chain staining. In LCDD, the pattern of glomerular immunofluorescence shows a marked heterogeneity. In contrast, tubular deposits of light chains are clearly visible and are seen in all cases [21]. We could easily have diagnosed this patient with LCDD at renal biopsy if light-chain staining had been performed, despite absent glomerular findings typical of LCDD on light microscopy. LCDD could also be diagnosed if electron microscopy had been done. Considering patient age and good general condition, high-dose therapy with PBSCT support would have been a good indication after the diagnosis of LCDD at the time of renal biopsy.

In conclusion, we experienced an instructive case of tubulointerstitial nephritis with poorly recognized pattern of renal disease associated with LCDD, which was followed by accelerated and massive light-chain deposition in glomeruli. This pattern is relatively rare, but it should be recognized as an initial manifestation of LCDD, and adequate treatment should be administered. A high index of suspicion is necessary to make the correct diagnosis, and routine $\kappa$ - and $\lambda$-light-chain staining and, if possible, electron microscopic examination are recommended in biopsied cases.

Acknowledgments The authors thank Kiyomi Kisu and Shuichi Futatsumori for their excellent technical assistance.

Conflict of interest The authors report no conflicts of interest.

\section{References}

1. Ronco PM, Alyanakian MA, Mougenot B, Aucouturier P. Light chain deposition disease: a model of glomerulosclerosis defined at the molecular level. J Am Soc Nephrol. 2001;12:1558-65.

2. Lin J, Markowitz GS, Valeri AM, Kambham N, Sherman WH, Appel GB, et al. Renal monoclonal immunoglobulin deposition disease: the disease spectrum. J Am Soc Nephrol. 2001;12: 1482-92.

3. Buxbaum J, Gallo G. Nonamyloidotic monoclonal immunoglobulin deposition disease. Light-chain, heavy-chain, and lightand heavy-chain deposition diseases. Hematol Oncol Clin North Am. 1999;13:1235-48.

4. Ganeval D, Noël LH, Preud'homme JL, Droz D, Grünfeld JP. Light-chain deposition disease: its relation with AL-type amyloidosis. Kidney Int. 1984;26:1-9.

5. Herrera GA. Renal manifestations of plasma cell dyscrasias: an appraisal from the patients' bedside to the research laboratory. Ann Diagn Pathol. 2000;4:174-200.

6. Gu X, Herrera GA. Light-chain-mediated acute tubular interstitial nephritis: a poorly recognized pattern of renal disease in patients with plasma cell dyscrasia. Arch Pathol Lab Med. 2006;130: 165-9.

7. Racusen L, Kashgarian M. Ischemic and toxic acute tubular injury and other ischemic renal injury. In: Jennette JC, Olson JL, Schwartz MM, Silva FG, editors. Heptinstall's pathology of the kidney. Philadelphia: Lippincott Williams \& Wilkins; 2007. p. 1139-98. 
8. Kapur U, Barton K, Fresco R, Leehey DJ, Picken MM. Expanding the pathologic spectrum of immunoglobulin light chain proximal tubulopathy. Arch Pathol Lab Med. 2007;131: 1368-72.

9. Gu X, Barrios R, Cartwright J, Font RL, Truong L, Herrera GA. Light chain crystal deposition as a manifestation of plasma cell dyscrasias: the role of immunoelectron microscopy. Hum Pathol. 2003;34:270-7.

10. Ma CX, Lacy MQ, Rompala JF, Dispenzieri A, Rajkumar SV, Greipp PR, et al. Acquired Fanconi syndrome is an indolent disorder in the absence of overt multiple myeloma. Blood. 2004;104:40-2.

11. Thorner PS, Bédard YC, Fernandes BJ. Lambda-light-chain nephropathy with Fanconi's syndrome. Arch Pathol Lab Med. 1983;107:654-7.

12. Lajoie G, Leung R, Bargman JM. Clinical, biochemical, and pathological features in a patient with plasma cell dyscrasia and Fanconi syndrome. Ultrastruct Pathol. 2000;24:221-6.

13. Preud'homme JL, Morel-Maroger L, Brouet JC, Cerf M, Mignon F, Guglielmi P, et al. Synthesis of abnormal immunoglobulins in lymphoplasmacytic disorders with visceral light chain deposition. Am J Med. 1980;69:703-10.

14. Venkataseshan VS, Faraggiana T, Hughson MD, Buchwald D, Olesnicky L, Goldstein MH. Morphologic variants of light-chain deposition disease in the kidney. Am J Nephrol. 1988;8:272-9.
15. Randall RE, Williamson WC Jr, Mullinax F, Tung MY, Still WJ. Manifestations of systemic light chain deposition. Am J Med. 1976;60:293-9.

16. Pozzi C, Locatelli F. Kidney and liver involvement in monoclonal light chain disorders. Semin Nephrol. 2002;22:319-30.

17. Heilman RL, Velosa JA, Holley KE, Offord KP, Kyle RA. Longterm follow-up and response to chemotherapy in patients with light-chain deposition disease. Am J Kidney Dis. 1992;20:34-41.

18. Royer B, Arnulf B, Martinez F, Roy L, Flageul B, Etienne I, et al. High dose chemotherapy in light chain or light and heavy chain deposition disease. Kidney Int. 2004;65:642-8.

19. Harada K, Akai Y, Sakan H, Yamaguchi Y, Nakatani K, Iwano $\mathrm{M}$, et al. Resolution of mesangial light chain deposits 3 years after high-dose melphalan with autologous peripheral blood stem cell transplantation. Clin Nephrol. 2010;74:384-8.

20. Firkin F, Hill PA, Dwyer K, Gock H. Reversal of dialysisdependent renal failure in light-chain deposition disease by autologous peripheral blood stem cell transplantation. Am J Kidney Dis. 2004;44:551-5.

21. Ronco P, Plaisier E, Mougenot B, Aucouturier P. Immunoglobulin light (heavy)-chain deposition disease: from molecular medicine to pathophysiology-driven therapy. Clin J Am Soc Nephrol. 2006;1:1342-50. 